\section{Milbenallergie: Erfolg mit neuen SLIT-Tabletten}

Täglich eingenommene Tabletten zur sublingualen Immuntherapie (SLIT) lindern bei Milbenallergikern bereits nach 14 Tagen Symptome der allergischen Rhinitis. Das bestätigen die Ergebnisse einer randomisierten placebokontrollierten Doppelblindstudie, in der zwei verschiedene Dosierungen geprüft wurden.

\begin{abstract}
An der Phase-III-Studie an 100 ZentAren in zwölf europäischen Ländern nahmen 992 Patienten im Alter zwischen 18 und 65 Jahren teil, die - trotz Pharmakotherapie - eine moderate bis schwere, durch Milbenallergene ausgelöste allergische Rhinitis hatten. Etwa ein Jahr lang wurden die Patienten mit hochstandardisierten SQ-HDM-SLIT(„,standardized quality house dust mite sublingual immunotherapy")-Tabletten in der Dosierung 6-SQ oder 12-SQ täglich oder mit einem Scheinpräparat behandelt. Die Tabletten enthielten die gleichen Mengen der Major-Antigene Der p 1, Der f 1, Der p 2 und Der $\mathrm{f} 2$ der Milbenarten Dermatophagoides pteronyssinus und farinae.

Primärer Endpunkt der Studie war eine Reduktion des durchschnittlichen Total Combined Rhinitis Score (TCRS), gemit-
\end{abstract}

telt über die letzten acht Wochen der Behandlung. Der Score (0 bis 24) berücksichtigt tägliche Rhinitissymptome und Medikation. Der Erfolg der Behandlung wurde zwischen 1. Oktober und 15. März beurteilt, also im europäischen Herbst und Winter, um Einflüsse durch eine mögliche Pollenallergie auszuschließen.

Durch die SLIT wurde im Vergleich zur Placebogruppe eine signifikante absolute Reduktion des TCRS erzielt, und zwar sowohl mit der 6-SQ-Dosis (um 1,18; $95 \%$-Konfidenzintervall $0,45-1,91 ; \mathrm{p}=$ 0,002 ) als auch mit der 12-SQ-Dosis (um 1,22; 95\%-Konfidenzintervall 0,49-1,96; $p=0,001)$. Als klinisch relevanter Unterschied zum Effekt unter Placebo war zuvor eine Differenz von 1 definiert worden. Die statistisch signifikante Differenz zu Placebo wurde in der von dem dänischen
Unternehmen ALK unterstützten Studie bereits nach 14 Tagen erreicht.

Außer der Symptomreduktion wurde durch die SLIT auch der Medikamentenbedarf signifikant reduziert, und zwar im Vergleich zu Placebo mit der niedrigeren Dosis um 0,69 Score-Punkte ( $\mathrm{p}=0,008)$ und mit der höheren Dosis um 0,60 Punkte $(\mathrm{p}=0,024)$.

Insgesamt betrachtet wurde die Immuntherapie gut vertragen. Die milden unerwünschten Wirkungen waren meist lokale allergische Reaktionen, die in den ersten Tagen auftraten und nach Tagen oder Wochen wieder verschwanden. Am häufigsten waren oraler Pruritus (20\%), Irritationen im Rachenraum (14\%) und Ödeme in der Mundhöhle (8\%).

Fazit: Wirkung und Sicherheit der beiden SLIT-Dosierungen konnten bestätigt werden.

Peter Leiner

Demoly $P$ et al. Effective treatment of house dust mite-induced allergic rhinitis with 2 doses of the SQ HDM SLIT-tablet: Results from a randomized double-blind, placebo-controlled phase III trial. J Allergy Clin Immunol 2015; doi: 10.1016/j.jaci.2015.06.036

\title{
Junge Patienten mit Schilddrüsenkarzinom: Zervikale LK-Metastasen beeinflussen Prognose
}

\section{Die Prognose bei jüngeren Patienten mit papillären Schilddrüsenkarzinomen galt bisher als gut, ungeachtet ihres lokoregionären Lymphknotenstatus. Doch man sollte dies differenzierter beurteilen.}

E in papilläres Schilddrüsenkarzinom — (PTC) bei Patienten unter 45 Jahren wird bisher als Niedrig-Risiko-Erkrankung eingestuft - auch wenn die Erkrankung lokoregionär fortgeschritten ist. In den aktuellen Stagingleitlinien des American Joint Committee on Cancer (AJCC) werden bei diesen Patienten die Krankheitsstadien lediglich entsprechend dem Vorliegen von Fernmetastasen unterschieden, was dem Stadium II entspricht, Patienten mit zervikalen Lymphknoten (LK)-Metastasen haben demnach eine Stadium-I-Erkrankung. Ein Grund für die bisherigen Empfehlungen ist wohl das Fehlen von aussagekräftigen Daten, u. a. zum Lymphknotenstatus, für die Prognose dieser Patientengruppe.
Das wurde nun mit einer Studie nachgeholt, in der Krankenakten von 1998 bis 2006 aus der National Cancer Data Base (NCDB) und dem Programm "Surveillance, Epidemiology, and End Results" (SEER) extrahiert wurden. Identifiziert wurden Patienten unter 45 Jahren, die wegen eines PTC im Stadium I operiert worden waren. Die NCDB steuerte 47.902 Patienten bei (11.740 mit und 36.162 ohne LK-Metastasen), die SEERDatenbank 21.855 Patienten (5.188 mit und 16.667 ohne positiven Nodalstatus). Nach Adjustierung zeigte sich bei Patienten mit zervikalen LK-Metastasen ein geringeres Gesamtüberleben als bei Patienten ohne lokoregionären LK-Befall (NCDB: Hazard Ratio [HR] 1,32;
$95 \%$-Konfidenzintervall [95\%-KI] 1,041,67; $\mathrm{p}=0,021$; SEER: HR 1,29; $95 \%$-KI $1,08-1,56 ; p=0,006$ ). Eine zunehmende Zahl metastasierter LK war mit einem sinkenden Gesamtüberleben assoziiert (HR 1,12; 95\%-KI 1,01-1,25; $\mathrm{p}=0,03$ ), erst bei $\geq 6$ positiven LK erhöhte sich das Risiko nicht mehr weiter (HR 0,99; $95 \%$-KI 0,99-1,05; $\mathrm{p}=0,75)$.

Fazit: Auch Patienten unter 45 Jahren mit einem papillären Stadium-I-Schilddrüsenkarzinom sollten entsprechend der Anzahl zervikal metastasierter Lymphknoten prognostisch stratifiziert werden. Bei lokoregionärem LK-Befall nimmt das Gesamtüberleben proportional zur Anzahl der positiven Lk ab, bei $6 \mathrm{LK}$ wird ein Plateau erreicht. Diese Erkenntnisse sollten nun in neue Leitlinien eingearbeitet werden.

Barbara Kreutzkamp

Adam MA et al. Presence and number of lymph node metastases are associated with compromised survival for patients younger than age 45 years with papillary thyroid cancer J Clin Oncol. 2015;33(21):2370-5 